\title{
Influence of dietary pattern, physical activity, and I148M PNPLA3 on steatosis severity in at-risk adolescents
}

\author{
Valerio Nobili • Daniela Liccardo • Giorgio Bedogni • \\ Guglielmo Salvatori · Daniela Gnani · Iliana Bersani · \\ Anna Alisi · Luca Valenti · Massimiliano Raponi
}

Received: 13 January 2014/ Accepted: 26 February 2014/Published online: 14 March 2014

(C) Springer-Verlag Berlin Heidelberg 2014

\begin{abstract}
Evidence relating dietary patterns to obesity and related disorders such as non-alcoholic fatty liver disease (NAFLD) is limited in pediatric age. Aim of this study was to analyze the association between dietary patterns, obesity and development of severe steatosis and the metabolic syndrome in a series of children and adolescents referred for suspected NAFLD, and the interaction with the rs738409 I148M PNPLA3 polymorphism. Two hundred patients (112 females) had completed a food frequency and demographic questionnaire. Nearly $58 \%$ were obese, and $32 \%$ were overweight. Mild, moderate, and severe fatty liver was present in $60(30 \%), 87(44 \%)$, and $51(26 \%)$ participants, respectively. A great proportion of overweight/obese children and adolescents reported a correct dietary pattern. At multivariate ordinal regression analysis considering demographic, anthropometric, genetic, and behavioral determinants, the major determinant of steatosis
\end{abstract}

Valerio Nobili and Daniela Liccardo have contributed equally to this work.

Electronic supplementary material The online version of this article (doi:10.1007/s12263-014-0392-8) contains supplementary material, which is available to authorized users.

V. Nobili $(\bowtie) \cdot$ D. Liccardo

Unit of Hepato-Metabolic Diseases, Bambino Gesù Children's

Hospital, IRCCS, Piazza S. Onofrio 4, 00165 Rome, Italy

e-mail: nobili66@yahoo.it

G. Bedogni

Clinical Epidemiology Unit, Liver Research Center, Trieste, Italy

G. Salvatori · I. Bersani

Neonatal Intensive Care Unit, Department of Medical and Surgical Neonatology, Bambino Gesù Children's Hospital, IRCCS, Rome, Italy severity was PNPLA3 I148M genotype $(p<0.0001)$, followed by older age $(p=0.017)$, higher waist circumference $(p=0.016)$, and less time spent practising physical exercise $(p=0.034)$. Furthermore, there was a significant interaction between PNPLA3 I148M and intake of sweetened beverages $(p=0.033)$ and of vegetables $(p=0.038)$. In conclusion, although dietary pattern was reportedly correct in at-risk overweight adolescents with NAFLD, we report a novel interaction between PNPLA3 I148M and dietary components with the severity of steatosis.

Keywords Food frequency - Dietary pattern - Nonalcoholic fatty liver disease $\cdot$ Obesity

\section{Introduction}

Obesity has become one of the major health problems worldwide affecting both children and adolescents. The growing prevalence of childhood obesity and overweight in industrialized countries is leading to a parallel surge in the prevalence of pediatric non-alcoholic fatty liver disease (NAFLD) (Vajro et al. 2012; Alisi et al. 2012; Loomba et al. 2009). Pediatric NAFLD is not always a benign

D. Gnani · A. Alisi

Liver Research Unit, Bambino Gesù Children's Hospital and Research Institute, Rome, Italy

L. Valenti $(\square)$

Department of Pathophysiology and Transplantation, Università degli Studi di Milano, Internal Medicine, Fondazione IRCCS Ca' Granda, Ospedale Maggiore Policlinico, Via F. Sforza 35, 20122 Milan, Italy

M. Raponi

Bambino Gesù Children's Hospital, IRCCS, Rome, Italy 
condition, with some children progressing to steatohepatitis (NASH) and fibrosis, with possible evolution to cirrhosis and liver failure (Loomba et al. 2009; Feldstein et al. 2009; Nobili et al. 2013). NAFLD affects $3-13 \%$ of children and adolescents, and this figure increases up to approximately $80 \%$ among obese individuals (Feldstein et al. 2009; Nobili et al. 2013). These children are at lifetime risk of developing metabolic complications such as dyslipidemia, insulin resistance, hypertension, and type 2 diabetes, as well as other features of the metabolic syndrome (Vajro et al. 2012; Alisi et al. 2009, 2012; Loomba et al. 2009; Feldstein et al. 2009; Nobili et al. 2013). During adolescence, children start following a personalized lifestyle characterized by excessive caloric intake, and sedentary lifestyle. All these changes predispose to the development of pediatric NAFLD by increasing in the body mass index (BMI) and visceral adiposity, and by decreasing physical fitness (Anitha Rani and Sathiyasekaran 2013).

To date, it is not known whether specific dietary patterns predispose to NAFLD independently of BMI or whether diet may protect against the development of the NAFLD (Anitha Rani and Sathiyasekaran 2013; Valenti et al. 2010a). Excessive consumption of simple sugars has been reported to promote the development of NAFLD in adults. A prospective association between dietary pattern, obesity, and metabolic complications has been reported by several studies in children (Johnson et al. 2007a, b, 2008; Ambrosini et al. 2012; Zelber-Sagi et al. 2007). However, evidence relating dietary patterns to NAFLD is limited in the pediatric age. Conversely, as suggested by different epidemiological and family studies, hereditary factors may play an essential role in determining the susceptibility to the development of NAFLD (Dongiovanni et al. 2013; Schwimmer et al. 2009; Guerrero et al. 2009; Makkonen et al. 2009).

The strongest genetic risk factor for NAFLD is by far the I148M variant of patatin-like phospholipase domaincontaining 3 (PNPLA3; encoded by rs738409 $\mathrm{C}>\mathrm{G}$ singlenucleotide polymorphism). The I148M variant influences liver fat without independently of body mass index, dyslipidemia, or insulin resistance, and it has been associated with severe steatosis, NASH, and liver fibrosis (Valenti et al. 2002, 2006, 2010a, b; Miele et al. 2008; Dongiovanni et al. 2010), even if severe obesity exposed an association between I148M, insulin resistance, and lower triglycerides in Northern European subjects (Krarup et al. 2012; Palmer et al. 2012). We previously showed that in overweight children with increased liver enzymes, the PNPLA3 I148M polymorphism is enriched compared to the general population (homozygosity for the $148 \mathrm{M}$ risk allele was 16 vs. $5 \%$ in the general population) and represents a non-invasive early marker of NASH and fibrosis (Valenti et al. 2010a).
Aim of this study was to analyze the association between dietary patterns, obesity and development of severe steatosis and the metabolic syndrome in a large series of children and adolescents with hepatic steatosis at ultrasonography, and the interaction with the rs738409 I148M PNPLA3 polymorphism.

\section{Patients and methods}

\section{Patients}

This prospective study included 200 consecutive obese children and adolescents (112 females) who were referred to the Bambino Gesù Children's Hospital (Rome, Italy) between January 2013 and June 2013 for metabolic alterations and suspected NAFLD, and underwent hepatological evaluation. Not all the patients were Caucasian of Italian descent. Two hundred patients had completed, with their primary caregivers, a food frequency (FFQ) and demographic (job, exercise habits, education and socio-economic status, etc.) questionnaire. We decided to classify the dietary habits of our children on the basis of food eaten every day or never in a week as it is difficult to assess food intake in terms of frequency in children. To obtain more accurate information, the consumption of a given food was coded as follows: 'every day of the week,' 'sometimes,' and 'never.' The questionnaire comprised numerous subsections (breakfast, morning snack, lunch, afternoon snack, dinner, etc.) that investigated the consumption of specific foods (Italian and international foods). As for food, the questionnaire comprised questions on physical activity performed during free time and systematic physical activity (sport type and frequency). The frequency of a given activity was coded as follows: 'four times or every day of the week,' ' $1-3$ times of the week,' and 'one time for month o less.'

Anthropometry, biochemistry, and radiological evaluation

Anthropometrical indices such as height (in $\mathrm{cm}$ ) and weight (in $\mathrm{kg}$ ) were assessed in all children using standardized methods. Waist circumference (WC) was measured taking the average of two measurements at the level of the umbilicus to the nearest $0.1 \mathrm{~cm}$ using a regular tape measure. BMI was calculated, and their corresponding BMI percentiles were calculated from the US Centers for Disease Control (CDC) and Prevention growth charts. Children were also classified as normal weight $(\geq 5 \%$ and $<85 \%)$, overweight $(\geq 85 \%$ and $<95 \%)$, and obese ( $\geq 95 \%$ ), according to the guidelines from the CDC (2013). A 2-h oral glucose tolerance test was performed following 
the recommendations of the World Health Organisation (2006). Glucose was measured by standard methods and insulin by means of radioimmunoassay (MYRIA Technogenetics, Milan, Italy). Liver enzymes were determined by standard methodology. Ultrasonographic evaluation was carried out by a pediatric radiologist with an experience in gastrointestinal US. The frequency of transducer for microconvex and linear (General Electric Logic 9) array was 5-8 and 10-14 MHz, respectively (Shannon et al. 2011). Other causes of hepatic steatosis (including alcohol abuse, drugs, and genetic diseases if clinically indicated) were excluded in each patient.

Patients selection critical for data interpretation

A total of 200 children aged $10-13$ years (112 females) participated in the study between January 2013 and June 2013. Of the 200 study participants, 187 were born in Italy, 137 (68 \%) Central Italy, 48 (24 \%) Southern Italy, 2 (1\%) Northern Italy, and 11 (6\%) outside of Italy. Two patients had not known the birthplace. The overall characteristics of study participants are presented in Table 1. One hundred and fourteen (57\%) were born from spontaneous delivery, $146(73 \%)$ were breastfed, and almost all of them (58\%) were exclusively breastfed. A positive history of allergy or other comorbidities were self-reported only by $25 \%$ of the children.

\section{Statistical analysis}

Steatosis severity determinants were evaluated at univariate and multivariate ordinal logistic regression analysis. We employed an additive genetic model to test the effect of the PNPLA3 variant (Valenti et al. 2010c; Sookoian and Pirola 2011). Given the striking association between PNPLA3 and ultrasonographic severity of steatosis, we kept in the multivariate model variables that were significantly associated with steatosis severity after correction for PNPLA3 I148M. We also tested for the interaction between PNPLA3 I148M, physical activity, and dietary components. $p$ values were considered significant when $<0.05$ (two-tailed).

\section{Results}

Anthropometric and biochemical profile

The anthropometric and serum biochemistry profile of study participants at the time of evaluation are shown in Tables 1, 2. Among the participants, $116(58 \%)$ were obese, 63 (32\%) were overweight, and only 21 (10\%)
Table 1 Metabolic parameters

\begin{tabular}{llll}
\hline$n=200$ & $P_{50}$ & $P_{25}$ & $P_{75}$ \\
\hline Age (years) & 11 & 10 & 13 \\
Weight (kg) & 54 & 42 & 66 \\
Weight (SDS) & 1.58 & 1.01 & 2.22 \\
Height (m) & 1.48 & 1.35 & 1.56 \\
Height (SDS) & 0.38 & -0.34 & 1.10 \\
BMI (kg/m $\left.{ }^{2}\right)$ & 25.1 & 22 & 27.4 \\
BMI (SDS) & 1.68 & 1.24 & 2.04 \\
Waist (cm) & 89 & 80 & 94 \\
Systolic BP (mmHg) & 109 & 102 & 118 \\
Diastolic BP (mmHg) & 70 & 61 & 74 \\
Cholesterol (mg/dl) & 160 & 136 & 185 \\
HDL (mg/dl) & 54 & 38 & 69 \\
Triglycerides (mg/dl) & 90 & 72 & 128 \\
Glucose at 0 min (mg/dl) & 82 & 75 & 90 \\
Glucose at 30 min (mg/dl) & 131 & 117 & 147 \\
Glucose at 60 min (mg/dl) & 143 & 122 & 165 \\
Glucose at 90 min (mg/dl) & 129 & 111 & 146 \\
Glucose at 120 min (mg/dl) & 116 & 100 & 130 \\
Insulin at 0 min (mU/l) & 11 & 7 & 16 \\
Insulin at 30 min (mU/l) & 90 & 66 & 132 \\
Insulin at 60 min (mU/l) & 104 & 57 & 134 \\
Insulin at 90 min (mU/l) & 99 & 48 & 142 \\
Insulin at 120 min (mU/l) & 92 & 47 & 129 \\
HOMA & 2.2 & 1.4 & 3.3 \\
QUICKI & 0.15 & 0.14 & 0.16 \\
IncAUC Insulin/IncAUC Glucose & 1.7 & 1.2 & 2.5 \\
ISI & 3.6 & 2 & 4.9 \\
ALT (U/l) & 70 & 50 & 87 \\
AST (U/l) & 49 & 39 & 64 \\
GGT (U/l) & 25 & 18 & 39 \\
\hline
\end{tabular}

were of normal weight as for the age and gender adjusted BMI percentiles of the WHO child growth standards. Large waist circumference was found in $176(88 \%)$ of the participants. No differences in alanine transaminase (ALT), aspartate transaminase (AST), gamma-glutamyltransferase (GGT), lipid profile, and all the other metabolic parameters were found between genders. Four $(2 \%)$ participants had all the features of metabolic syndrome while ninety-nine $(49.5 \%)$ participants had only one of these characteristics. Only $14 \%$ had all the criteria of the metabolic syndrome as shown in Table S1. At ultrasonographic evaluation, liver echostructure appeared normal in $2(1 \%)$ children, while liver parenchyma was more echogenic and coarse than normal in 198 (99\%) participants. Mild, moderate, and severe fatty liver was present in $60(30 \%), 87$ (44\%), and $51(26 \%)$ participants, respectively. 
Table 2 Characteristics of children enrolled

\begin{tabular}{lc}
\hline & Total $n=200$ \\
& $N(\%)$ \\
\hline Place & \\
North & $2(1.0)$ \\
Center & $137(68.5)$ \\
South & $48(24)$ \\
Outside Italy & $11(5.5)$ \\
Unknown & $2(1.0)$ \\
Gender & \\
Female & $112(56)$ \\
Male & $88(44)$ \\
Delivery & \\
Regular & $114(57)$ \\
Cesarean & $86(43)$ \\
Breastfed & \\
No & $52(26)$ \\
Yes & $146(73)$ \\
Exclusively breastfed & \\
No & \\
Yes & \\
Unknown & $112(40)$ \\
Self-reported disease & $51(26)$ \\
No & $50(25)$ \\
Yes & \\
Unknown & \\
Physical activity & \\
$4+$ times for week & \\
$1-3$ times for week & \\
\hline
\end{tabular}

Dietary behavior

We discuss here the most relevant data that emerged from the study of dietary habits (Table S2, S3).

As far as breakfast is concerned, $130(65 \%)$ of the children did eat it regularly (i.e. 'every day'), 68 (34\%) eating it irregularly (i.e. 'sometimes'), and 2 (1\%) not eating it at all (i.e. 'never'). Milk was eaten regularly at breakfast by almost all children ( $P$ ns). Morning and afternoon snacks were eaten regularly by $186(93 \%)$ and 187 (94\%) of participants, respectively. A morning snacks consisted mainly of crackers, crepes, pizza, and salty foods. Conversely, an evening snack consisted mainly of sweet products (or foods), such as biscuits and snacks. Approximately half of participants $103(52 \%)$ have eating habit between meals and only $10(5 \%)$ eats 'every day' between meals. Lunch and dinner were regularly eaten by 197 (99\%) of the children. Among the foods 'never' eaten, grated cheese was the most frequent (32\%), followed by yogurt $(22 \%)$ while different types of cheese were
Table 3 Variables significantly associated with the ultrasonographic severity of steatosis at univariate ordinal regression analysis in 200 overweight adolescents with suspected NAFLD

\begin{tabular}{llll}
\hline & Estimate & SE & $p$ value \\
\hline BMI centile & +0.08 & 0.02 & $4.0 \times 10^{-3}$ \\
WC centile & +0.10 & 0.03 & $5.6 \times 10^{-3}$ \\
Triglycerides (mg/dl) & +0.0004 & 0.0002 & $4.3 \times 10^{-2}$ \\
GGT (IU/l) & +0.015 & 0.007 & $3.2 \times 10^{-2}$ \\
I148M alleles & +4.32 & 0.50 & $5.0 \times 10^{-39}$ \\
\hline
\end{tabular}

'sometimes' eaten by $88 \%$ of participants. Among the foods 'every day' eaten, cereals were the most frequent $78 \%$, followed by vegetables (34\%), meat (33\%), cured meat $(22 \%)$, fruits $(18 \%)$, legumes $(4 \%)$, and fish and eggs ( $1 \%)$. Overall, $97 \%$ of participants ate fruit, such as oranges, apples, or bananas, one or more times per day. Ninety-seven percentage of participants usually ate vegetables, such as green salad, potatoes, or tomatoes, one or more times per day (Tables S2, S3). Seven percentage reported drinking sodas one or more times at mealtimes. Extra virgin oil was consumed by $87 \%$ of children. All other types of condiments, sauces, and dressings were 'never or rarely' eaten: olive oil (88\%), corn oil (98\%), peanut oil (99\%), sunflower oil (98\%), mixed seed oil (99\%), butter (96\%), margarine (98\%), and mayonnaise and ketchup (1\%). No difference regarding eating habits was found between genders $(P \mathrm{~ns})$.

Physical activity

About $82 \%$ of the children declared practicing a sport: gymnastics $(21 \%)$, swimming (19\%), soccer (11\%), basketball $(11 \%)$, or other (38\%). Seventy-four percentage practiced sport at least once per week (Table 2).

Severity of steatosis: anthropometric, metabolic, genetic, and environmental determinants

Demographic, anthropometric, metabolic, and environmental factors associated with steatosis severity are presented in Table 3. Severity of steatosis was associated with adiposity (BMI and WC centile), borderline associated with serum triglycerides and GGT levels, and strongly influenced by PNPLA3 genotype $\left(p<10^{-36}\right.$; Fig. 1$)$. Clinical features according to PNPLA3 I148M genotype are presented in Table S4. Given the striking effect of PNPLA3 genotype on steatosis grade, we next tested the effect of environmental determinants of liver fat (physical activity and diet) at multivariate analysis adjusted for PNPLA3 and other confounding factors.

At multivariate ordinal regression analysis considering demographic, anthropometric, genetic, and behavioral 


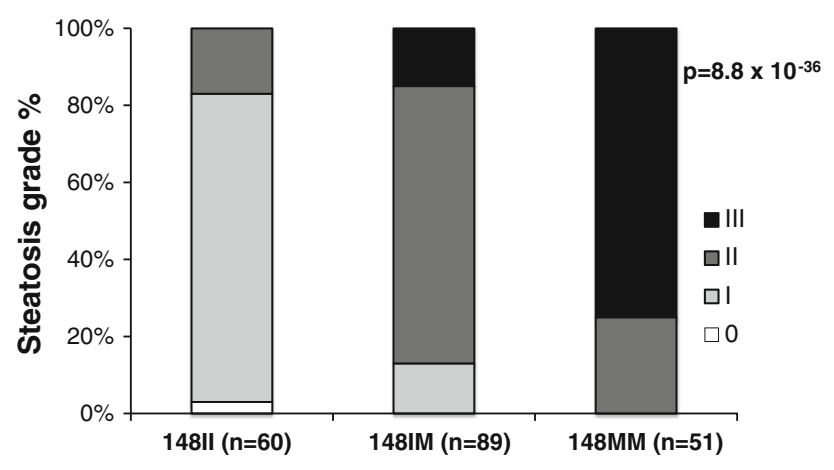

Fig. 1 PNPLA3 I148M genotype

Table 4 Independent predictors of ultrasonographic severity of steatosis in 200 overweight adolescents with suspected NAFLD

\begin{tabular}{llll}
\hline & Estimate & SE & $p$ value \\
\hline Age (years) & +0.21 & 0.08 & $1.7 \times 10^{-2}$ \\
WC centile & +0.15 & 0.06 & $1.6 \times 10^{-2}$ \\
I148M alleles & +4.32 & 0.50 & $2.0 \times 10^{-18}$ \\
Physical exercise hours & -1.78 & 0.61 & $3.4 \times 10^{-3}$ \\
I148M $\times$ sodas & +0.76 & 0.35 & $2.3 \times 10^{-2}$ \\
I148M $\times$ vegetables & -1.31 & 0.62 & $3.4 \times 10^{-2}$ \\
\hline
\end{tabular}

$W C$ waist circumference

determinants of steatosis severity (Table 4), the major determinant of ultrasonographic severity of steatosis was PNPLA3 148M alleles $(p<0.0001)$. Steatosis severity was independently associated with older age $(p=0.017)$, higher WC centile $(p=0.016)$, and negatively associated with weekly hours of physical exercise $(p=0.034)$. Although the consumption of specific foods or dietary patterns were not directly associated with liver fat content, there was a significant interaction between PNPLA3 I148M alleles and moderate to severe intake of sweetened beverages, associated with induction of hepatic lipogenesis, in the severity of steatosis $(p=0.033)$, i.e. the $\mathrm{I} 148 \mathrm{M}$ mutations were more strongly associated with steatosis in those who reported drinking sweetened drinks at least once weekly. In addition, there was a significant interaction between I148M variant and intake of vegetables on steatosis severity ( $p=0.038$ ), i.e. the association of the I148M variant with steatosis severity was weaker in those who reported a vegetable-poor diet.

\section{Discussion and conclusion}

The present study reports on dietary behavior, levels of physical activity, the metabolic implications, and prevalence of obesity among a large series of Italian children and adolescents with fatty liver. The prevalence of risk factors for obesity is fairly high among the adolescents. This study shows that a great proportion of overweight/ obese children and adolescents had correct dietary habits (Vyncke et al. 2013) and confirm that genetic factors, and in particular the I148M variant of PNPLA3, play a major role in the development and severity of steatosis in obesity during the developmental age, representing by far the strongest determinant. These and previous data (Valenti et al. 2010b) suggest that severe steatosis and NASH behave similarly to a monogenic disease in obese individuals during the developmental age. Severity of abdominal fat and older age were other independent contributors, while we report for the first time a protective effect of the weekly time spent in physical exercise on steatosis severity. In the present cohort including only adolescents referred for suspected metabolic disease, we did not detect any interaction between PNPLA3 I148M and body fat in the pathogenesis of NAFLD. However, it could be speculated that the association between the $148 \mathrm{M}$ risk allele with higher BMI (as PNPLA3 I148M does not affect body fat at population level) is due to the interaction between this genetic factor and weight in determining higher ALT levels leading to patients referral (Romeo et al. 2010).

Although a higher intake of fast food, soft drinks, and meat has previously been associated with NAFLD in adults (Nobili et al. 2013), an association between dietary patterns, occurrence of obesity, and development of NAFLD has not been reported in pediatric age. However, an interaction between the I148M PNPLA3 variant and intake of carbohydrates has previously been reported in the pathogenesis of liver steatosis in Hispanic youth (Davis et al. 2012). In line with these previous results, we detected a significant interaction between I148M PNPLA3 and sweetened beverages, known to induce hepatic lipogenesis (Vos and Lavine 2013), on steatosis severity. In addition, the predisposing effect of I148M variant on steatosis severity was weaker in individuals consuming diets poor in vegetables. These data are in line with the hypothesis that the effect of the PNPLA3 I148M is higher for diets rich in carbohydrates supporting hepatic lipogenesis (Sevastianova et al. 2011). It could be speculated that vegetables represent a source of substrates for hepatic lipogenesis, but additional studies with more detailed questionnaires are required to confirm this hypothesis. In contrast, we did not find evidence of interaction between PNPLA3 I148M and foods rich in fatty acids (Nobili et al. 2013). These novel results need to be replicated in larger appropriately designed studies, and interventional studies need to be conducted, before personalized dietary pattern be advised according to individuals PNPLA3 genotype. 
Two major limitations of this study are the lack of assessment of portion sizes, and lack of a control population (children without metabolic risk factors), and the noninvasive estimation of the severity of steatosis by US without direct quantification by MRI or liver histology. Therefore, we could not evaluate the interaction of PNPLA3 with abdominal obesity. Finally, it needs to be highlighted that the submitted questionnaires are not yet validated and that results based on the evaluation of interaction between PNPLA3 I148M and acquired factors need to be independently replicated.

In conclusion, despite likely increased caloric intake, obese adolescents report an apparently almost correct dietary pattern (Vyncke et al. 2013). The severity of the hepatic complication resulting from insulin resistance, i.e. hepatic steatosis, as detected by abdominal ultrasonography, is influenced by age, abdominal fat, and in particular by the genetic makeup, i.e. the number of I148M PNPLA3 alleles carried. Though, confirming previous reports in adults, longer duration of regular physical exercise seems to play a protective role, although a cause-effect relationship cannot be established based on the cross-sectional design of this study.

Interestingly, we report an interaction between dietary components, i.e. intake of sweetened beverages and of vegetables, and PNPLA3 I148M with the severity of steatosis, suggesting that evaluation of PNPLA3 and other genetic factors may help advising specific diets tailored on the specific individual. There is a need for further investigation as whether there is a causal relationship between the I148M PNPLA3 variant, dietary pattern, and development of pediatric NAFLD.

Conflict of interest Valerio Nobili, Daniela Liccardo, Giorgio Bedogni, Guglielmo Salvatori, Daniela Gnani, Iliana Bersani, Anna, Alisi, Luca Valenti, and Massimiliano Raponi declare that they have no conflict of interest.

Ethical standard The study was approved by the Ethical Committee of the Ospedale Bamin Gesù Roma and was conducted according to principles of the Declaration of Helsinki. Each subject or guardian of child included in the study signed a written informed consent.

\section{References}

Alisi A, Manco M, Vania A et al (2009) Pediatric nonalcoholic fatty liver disease in 2009. J Pediatr 155:469-474

Alisi A, Feldstein AE, Villani A et al (2012) Pediatric nonalcoholic fatty liver disease: a multidisciplinary approach. Nat Rev Gastroenterol Hepatol 9:152-161

Ambrosini GL, Emmett PM, Northstone K et al (2012) Identification of a dietary pattern prospectively associated with increased adiposity during childhood and adolescence. Int $\mathrm{J}$ Obes 36:1299-1305
Anitha Rani M, Sathiyasekaran BWC (2013) Behavioural determinants for obesity: a cross-sectional study among urban adolescents in India. Prev Med Public Health 46:192-200

Centers for Disease Control and Prevention. About BMI for children and teens [cited 2013 Jul 13]. http://www.cdc.gov/healthy weight/assessing/bmi/childrens_bmi/about_childrens_bmi.html

Davis JN, Whaley SE, Goran MI (2012) Effects of breastfeeding and low sugar-sweetened beverage intake on obesity prevalence in Hispanic toddlers. Am J Clin Nutr 95(1):3-8

Dongiovanni P, Valenti L, Rametta R et al (2010) Genetic variants regulating insulin receptor signaling are associated with the severity of liver damage in patients with nonalcoholic fatty liver disease. Gut 59:267-273

Dongiovanni P, Anstee QM, Valenti L (2013) Genetic predisposition in NAFLD and NASH: impact on severity of liver disease and response to treatment. Curr Pharm Des 19(29):5219-5238

Feldstein AE, Charatcharoenwitthaya P, Treeprasertsuk S et al (2009) The natural history of non-alcoholic fatty liver disease in children: a follow-up study for up to 20 years. Gut 58:15381544

Guerrero R, Vega GL, Grundy SM et al (2009) Ethnic differences in hepatic steatosis: an insulin resistance paradox? Hepatology 49:791-801

Johnson L, Mander AP, Jones LR et al (2007a) Is sugar-sweetened beverage consumption associated with increased fatness in children? Nutrition 23:557-563

Johnson L, Mander AP, Jones LR et al (2007b) A prospective analysis of dietary energy density at age 5 and 7 years and fatness at 9 years among UK children. Int J Obes 32:586-593

Johnson L, Mander AP, Jones LR et al (2008) Energy-dense, lowfiber, high-fat dietary pattern is associated with increased fatness in childhood. Am J Clin Nutr 87:846-854

Krarup NT, Grarup N, Banasik K et al (2012) The PNPLA3 rs738409 G-allele associates with reduced fasting serum triglyceride and serum cholesterol in Danes with impaired glucose regulation. PLoS One 7:e40376

Loomba R, Sirlin CB, Schwimmer JB et al (2009) Advances in pediatric nonalcoholic fatty liver disease. Hepatology 50:12821293

Makkonen J, Pietilainen KH, Rissanen A et al (2009) Genetic factors contribute to variation in serum alanine aminotransferase activity independent of obesity and alcohol: a study in monozygotic and dizygotic twins. J Hepatol 50:1035-1042

Miele L, Beale G, Patman G et al (2008) The Kruppel-like factor 6 genotype is associated with fibrosis in nonalcoholic fatty liver disease. Gastroenterology 135:282-291

Nobili V, Svegliati-Baroni G, Alisi A et al (2013a) A 360-degree overview of paediatric NAFLD: recent insights. J Hepatol 58(6): $1218-1229$

Nobili V, Bedogni G, Donati B et al (2013b) The I148M variant of PNPLA3 reduces the response to docosahexaenoic acid in children with non-alcoholic fatty liver disease. J Med Food 16(10):957-960

Palmer CN, Maglio C, Pirazzi C et al (2012) Paradoxical lower serum triglyceride levels and higher type 2 diabetes mellitus susceptibility in obese individuals with the PNPLA3 148M variant. PLoS One 7:e39362

Romeo S, Sentinelli F, Dash S et al (2010) Morbid obesity exposes the association between PNPLA3 I148M (rs738409) and indices of hepatic injury in individuals of European descent. Int J Obes (Lond) 34:190-194

Schwimmer JB, Celedon MA, Lavine JE et al (2009) Heritability of nonalcoholic fatty liver disease. Gastroenterology 136:15851592

Sevastianova K, Kotronen A, Gastaldelli A et al (2011) Genetic variation in PNPLA3 (adiponutrin) confers sensitivity to weight 
loss-induced decrease in liver fat in humans. Am J Clin Nutr 94(1):104-111

Shannon A, Alkhouri N, Carter-Kent C et al (2011) Ultrasonographic quantitative estimation of hepatic steatosis in children With NAFLD. J Pediatr Gastroenterol Nutr 53:190-195

Sookoian S, Pirola CJ (2011) Targeting the renin-angiotensin system: potential beneficial effects of the angiotensin II receptor blockers in patients with nonalcoholic steatohepatitis. Hepatology 54(6): 2276-2277

Vajro P, Lenta S, Socha P et al (2012) Diagnosis of nonalcoholic fatty liver disease in children and adolescents: position paper of the ESPGHAN Hepatology Committee. J Pediatr Gastroenterol Nutr 54:700-713

Valenti L, Fracanzani AL, Dongiovanni P et al (2002) Tumor necrosis factor alpha promoter polymorphisms and insulin resistance in nonalcoholic fatty liver disease. Gastroenterology 122:274-280

Valenti L, Dongiovanni P, Piperno A et al (2006) Alpha1-antitrypsin mutations in NAFLD: high prevalence and association with altered iron metabolism but not with liver damage. Hepatology 44:857-864

Valenti V, Alisi A, Galmozzi E et al (2010a) I148M Patatin-like phospholipase domain-containing 3 gene variant and severity of pediatric nonalcoholic fatty liver disease. Hepatology 52:12741280
Valenti L, Al-Serri A, Daly AK et al (2010b) Homozygosity for the PNPLA3/adiponutrin I148M polymorphism influences liver fibrosis in patients with nonalcoholic fatty liver disease. Hepatology 51:1209-1217

Valenti L, Alisi A, Galmozzi E et al (2010c) I148M patatin-like phospholipase domain-containing 3 gene variant and severity of pediatric nonalcoholic fatty liver disease. Hepatology 52(4): 1274-1280

Vos MB, Lavine JE (2013) Dietary fructose in nonalcoholic fatty liver disease. Hepatology 57(6):2525-2531

Vyncke K, Cruz Fernandez E, Fajó-Pascual M et al (2013) Validation of the Diet Quality Index for Adolescents by comparison with biomarkers, nutrient and food intakes: the HELENA study. Br J Nutr 109(11):2067-2078

World Health Organization (WHO), International Diabetes Federation (IDF). Definition and diagnosis of diabetes mellitus and intermediate hyperglycemia. Report of a WHO/IDF Consultation, 2006

Zelber-Sagi S, Nitzan-Kaluski D, Goldsmith R et al (2007) Long term nutritional intake and the risk for non-alcoholic fatty liver disease (NAFLD): a population based study. J Hepatol 47:711-717 